Maria Gabriella Buzzi

Received: 5 May 2006

Accepted in revised form: 27 May 2006

Published online: 15 June 2006

M. Gabriella Buzzi (更)

Headache Centre,

IRCCS Fondazione Santa Lucia,

Via Ardeatina 306, I-00179 Rome, Italy

e-mail:mg.buzzi@hsantalucia.it

Tel.: +39-06-515011

Fax: +39-06-51501752

\section{Reading data in migraine acute treatment studies: from clinical trials to reviews and meta-analyses}

\begin{abstract}
The huge amount of data regarding triptans in clinical practice provides a great opportunity to learn about their efficacy, tolerability and adverse events and offers to the clinician the background for the best treatment option in any patient, given that the patient is suitable for being treated with any of those compounds. A detailed clinical history is necessary in order to make a preliminary selection of triptan-suitable or -unsuitable migraine patients. Among the former, a secondary selection may be made, to recognise different attacks that can be successfully
\end{abstract}

treated with different triptans, according to attack presentation and progression, within the same patient. Reading single original articles has become very difficult. Therefore, data are often presented in review form, which may be too partial or oriented. Systematic reviews or meta-analyses better serve to comment on data as they follow an analytic method that is based upon pooling data from original articles and comparing them using statistical aids.

Key words Migraine - Triptans • Clinical trial $\cdot$ Meta-analysis

\section{Introduction}

The increasing availability of molecules designed for targeted treatment of migraine attacks, the triptans, has produced a proliferation of data regarding the efficacy of such molecules in relieving migraine pain, accompanying symptoms and disability. The majority of clinical studies have been designed to evaluate the efficacy of each compound vs. placebo or vs. sumatriptan, the prototype, i.e., the gold standard, of the triptan class, and head-to-head studies between second-generation triptans are also available. The number of publications is outstanding and it would be difficult to go through all of them. Therefore, information is often sought by reading reviews that sum- marise data from original articles. However, such a timesaving learning method, may, somehow, drive the attention towards certain parameters and obscure other features, according to the purpose of a given Author and therefore provide partial information on a given issue. To obviate the risk of partial or driven information, the metaanalytic method has been proposed in order to revise and analyse data with statistical aids from large numbers of original trials and to provide objective conclusions.

The present paper should not be considered as a review as it will not take into consideration the whole literature concerning triptans, the author not being prone to such a titanic enterprise. Rather, it will make some examples of different ways of producing data and try to discuss the modalities of reading them to avoid misinterpretation and 
misuse of collected information. By definition, the citation of articles and compounds will not be comprehensive and will not report any specific result concerning the triptans, as this is not the aim of this commentary. The author denies any personal or institutional interest in proposing the following comments and reaffirms that "clinical practice should reflect scientific evidence blended with clinical experience and expertise" $[1,2]$.

\section{Clinical trials}

The design of clinical trials concerning triptans is basically very simple. Fig. 1 represents the simplified sketch of a trial and is based upon six parameters, all being mandatory when preparing the protocol:

\section{Randomisation}

It is necessary to assign the compounds in a casual manner to a sufficient number of patients, to avoid any prediction of what compound is assigned to a given patient, to maintain reliability if any patient will drop out for any reason during the study.

\section{Study design}

The study can be planned to compare two active drugs or an active drug to placebo; in placebo-controlled studies the patient usually receives two active doses and a placebo dose; both patient and researcher are blind to the content of any single dose preparation. In the triple-blind design, the monitor is also unaware of the content in each dose preparation. The researcher is authorised to open the randomisation code and verify the content of a dose prepa-

\section{Clinical trials in acute migraine treatment}

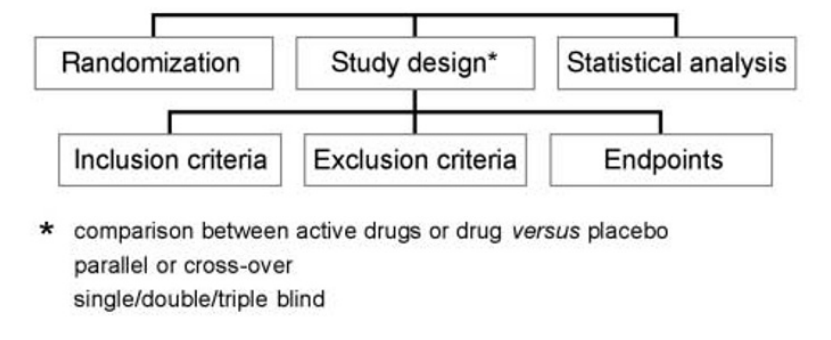

Fig. 1 Clinical trials in acute migraine treatment ration only in the presence of serious adverse events occurring during the study that are considered certainly or probably related to the study.

\section{Statistical analysis}

As a general rule, stat aids have to verify the probability that the event of obtaining success in treating a migraine attack is related to drug intake and is not casual. Data are analysed by direct comparison with no need of correction as no confounding factor is present if proper inclusion and exclusion criteria are followed (see below). The odds ratio (OR) is used to define the number of times the event has occurred and the number of times it has not. In migraine attack treatment trials the OR expresses the ratio between active drug-related event and placebo (or second active drug)-related event. The confidence interval (CI) for differences is usually assumed at $95 \%$ and expresses the range values in which $95 \%$ of events will fit when repeating the test.

\section{Inclusion criteria}

This parameter has been largely simplified, international diagnostic guidelines having been available since 1988 [3]. The issue of appropriate clinical diagnosis does not need further comment. Specific inclusion criteria have to be applied as general rules in order to reflect the general migraine population: (i) migraine history of at least 1 year; (ii) patient age from 18 to 65 years; (iii) presence of 1-6 attacks/month. The protocol should also include a criterion to warrant an adequate proportion of females and males in the study group. The latter criterion is not mandatory.

\section{Exclusion criteria}

A detailed clinical history of patients should be obtained to exclude those patients who are not eligible for the study, i.e., those patients in whom the study drug is contraindicated. Besides, other features have to be disclosed: (i) lateonset migraine ( $>50$ years); (ii) inability of the patient to differentiate a migraine attack from other headaches (most commonly from tension-type headache); (iii) inability to provide informed consent; (iv) inability or lack of compliance in providing an accurate diary of the attack; (v) use of other acute treatment compounds that are not allowed concomitantly to the study drug; (vi) risk of pregnancy. 


\section{Endpoints}

This is probably the most critical parameter when dealing with the available literature in this field. In fact, as a general rule, patients were instructed to treat their attack when pain was of moderate intensity. On a 4-point scale this is defined as a score of $2(0=$ absence of pain; $1=$ mild pain; $2=$ moderate pain; $3=$ severe pain), and within $6-8 \mathrm{~h}$ of pain onset. Initially, the response to drug was evaluated as an improvement of pain at $2 \mathrm{~h}$ post-dose [4], whereas the pain-free parameter at the same time point has been introduced more recently [5]. Other parameters to measure efficacy of study drug on pain, such as Pain Intensity Difference (PID) or Sum of Pain Intensity Difference (SPID), are not validated for trial purposes. It is intuitive that the results from studies that applied different efficacy measures are not comparable. This also applies when evaluating the efficacy of study compounds on accompanying symptoms. Also, the emerging "the sooner, the better" treatment option [6] makes the earlier trials less useful to define the efficacy of certain triptans. It is important to define the endpoints at the beginning of the study, namely the primary and the secondary endpoints, and set the statistical approach and the expected results. Following these criteria, the analysis will satisfy an ad hoc study design, i.e., a trial that will serve to verify the hypothesis that has been proposed. Post hoc analysis should be disregarded for educational purposes, as it is done to find out whether the study has produced other information, other than that for which the original trial was designed. In fact, those ancillary findings may not be applicable to the population studied, as patients were initially instructed to carefully obtain certain data and not others. Therefore, post hoc analysis might be partial and need further trials for testing and validating information according to well defined methods, endpoints and statistical analysis.

The guidelines for clinical trials [5] provide several recommendations on the above issues. Among them, the inclusion of placebo is strongly advised. Triptans should always be compared to placebo, and placebo controls should be also included when comparing more triptans with each other, due to the variability of responses to placebo (from $6 \%$ to $41 \%$ ). Referring to historical placebo effects is not suitable. The study should allow a rescue medication, and this should be permitted at $2 \mathrm{~h}$ post-dose, if necessary. Therefore, the measure of pain-free at $2 \mathrm{~h}$ post-dose should represent the primary endpoint for efficacy evaluation. Another issue concerns the incidence of relapse, previously named recurrence. It should be clear that the relapse applies only to those attacks in which pain was successfully treated (pain-free parameter) at $2 \mathrm{~h}$ postdose. Table 1 shows a simplified example to calculate relapse in a head-to-head comparison.

\section{Reviews and meta-analyses}

As stated above, reviews are sometimes a useful method for presenting a few studies within the same publication in order to focus attention on a certain drug. However, they simply represent a summary of available data and lack of methodology or discussion that may enrich the original data. As an example, Dodick [7] reported a series of papers regarding efficacy and tolerability of almotriptan. It is obvious that the studies cannot be pooled together, the endpoints being different in each trial and involving different methods: comparison with placebo, comparison with sumatriptan, long-term efficacy open study. Also, a post hoc analysis concerning the utility of early treatment (an endpoint that was not chosen prior to any trial indicated), is described. Ryan et al. [8] summarise data from 3 different studies on the clinical efficacy of frovatriptan $v s$. placebo. Besides having different endpoints, the studies were not available as published original articles and the Author refers to two abstract citations and to a dose-finding study. Geraud et al. [9] refers to 5 different clinical studies regarding frovatriptan. Among them there is a dose-finding study, 2 studies are $v s$. placebo, 1 is $v s$. placebo and sumatriptan $100 \mathrm{mg}$ (with open-label treatment for second and third attack) and 1 is a long-term open study. The adverse event incidence has been pooled from the different studies and reported as not different from placebo. Interestingly, Fisher's exact test, as per-

Table 1 How to calculate relapses

\begin{tabular}{lcc}
\hline & Compound A & Compound B \\
\hline Number of patients & 100 & 100 \\
Pain-free at 2 hours post-dose & $60 / 100$ & $40 / 100$ \\
Relapse & $20 / 60$ & $20 / 40$ \\
$\%$ Relapse & $33.3 \%$ & $50 \%$ \\
\hline
\end{tabular}

The number of relapses is the same in the two groups (compound A- and compound B-treated groups). However, the percent of relapses, calculated within each pain-free sub-population, is different, ant it may have statistical significance 
formed by Tfelt-Hansen and Steiner [10], shows that the incidence of side effects in the above studies is extremely high ( $p<0.00005$ when compared to placebo).

An attempt to apply methodology is made in a post hoc analysis of 5 different studies comparing rizatriptan with sumatriptan $(25,50 \mathrm{or} 100 \mathrm{mg})$ and naratriptan on the nausea symptom of migraine [11]. Although the trials share similar study design, the endpoints chosen prior to trials, namely relief of pain and associated symptoms, did not focus on the nausea itself and it is possible that patients did not report mild nausea (either as an accompanying symptom that has improved following attack treatment or as an adverse event to the treatment itself) after being pain-free.

As said above, systematic reviews and meta-analyses are of better use because they statistically analyse very large numbers of trials (hence, a very large number of attacks, either on active drug or placebo) to obtain:

- higher statistical power;

- better estimation of drug efficacy;

- population sub-groups;

- new hypotheses to be verified.

In 2001, Ferrari et al. [12] proposed a meta-analysis on 53 acute migraine treatment trials including 24,089 patients. All trials were randomised (placebo-controlled), cross-over and double blind. Cumulative data for sumatriptan $100 \mathrm{mg}$ (gold standard) and for all different triptans were obtained from international publications and registration packages (according to Good Clinical Practice method). The Authors declared no conflict of interest in their study, i.e., the study was not commissioned or sponsored by any pharmaceutical company. The latter declaration has an outstanding value in this context, as it means that the Authors qualified themselves as independent from any biased interpretation of the results.

The work takes many clinical parameters into consideration in order to propose the final list of triptans according to the best efficacy+tolerability profile. Efficacy parameters were:

- headache response at $2 \mathrm{~h}$ post-dose: pain reduction of at least two points in the "0-3" scale (see above), i.e., moderate pain to absence of pain, or severe pain to mild or absent;

- pain-free at $2 \mathrm{~h}$ post-dose;

- headache recurrence (or relapse);

- $\quad$ sustained pain-free (no recurrence within $24 \mathrm{~h}$, no need of rescue medication, no need of second dose);

- intra-individual consistency (i.e., the drug is consistently effective over multiple attacks);

- adverse events.

As said above, the effects of inactive compound (placebo) accounts for up to $41 \%$ of good responses. Therefore, an interesting way of presenting and compar- ing data is represented by the therapeutic gain (TG), which is calculated by subtracting the number of attacks that were successfully treated with placebo to the number of attacks that were successfully treated by active drug. The same applies to the therapeutic harm $(\mathrm{TH})$ regarding the occurrence of side effects due to placebo or active drug [13]. The reciprocal of those values is represented by the number needed to treat (NNT) and the number needed to harm $(\mathrm{NNH})$ values, which express the number of patients who have to receive the drug to obtain one successfully treated attack and the number of patients who have to be treated to get one side effect. It is intuitive that the best compound has a very low NNT and a very high NNH value. Accordingly, the best compound has the highest TG and the lower TH. Ferrari et al. [12] reported the data regarding the above clinical parameters using both the comparison between absolute values and the TG and $\mathrm{TH}$ values. Interestingly, the large number of patients reevaluated in this analysis make some issues even more evident and raises questions that need to be evaluated in more detailed fashion.

For instance, the definition of recurrence (or relapse) applies to pain that recurs within $24 \mathrm{~h}$. Usually subjects are instructed to treat pain when the intensity is moderate or severe. The question is: what to do if pain is mild (score 1 on a $0-3$ scale)? There are no indications to answer this question and maybe they will come from further studies regarding early treatment, as suggested by certain clinical

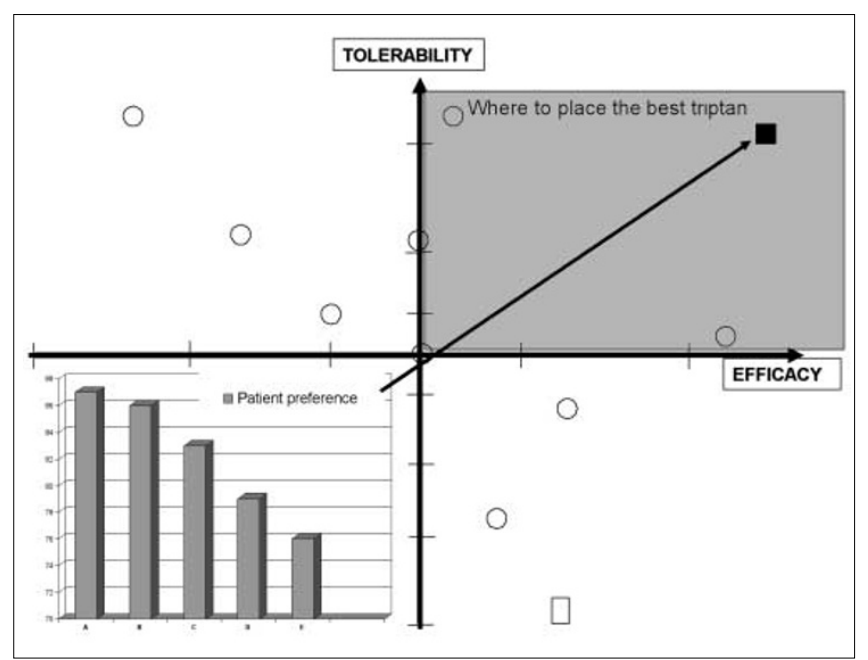

Fig. 2 Cross-linking tolerability and efficacy data is necessary for choosing the best triptan option and the "ideal triptan" (black box) should be located at the top level of tolerability and the highest value of efficacy. As no triptan has yet reached such a position, the choice should be made according to scientific data and to patient preference as well. The bottom histogram shows patient preference as reported by Lanteri-Minet et al. [15]: (A) pain free; (B) no relapse; (C) fast action; (D) no adverse events; (E) relief of accompanying symptoms 
studies [6]. Another issue concerns the consistency of response. The trend is that the percent of either the headache response and the pain-free decreases over the attacks, i.e., the percent efficacy is lower in the second and third attack treated. The placebo effect decreases even more in multiple attacks, therefore, the "placebo problem" is not solved, and it makes the across-patient and intrapatient variability still too high. Finally, the evaluation of adverse events should take into consideration the possibility that some of them are actually accompanying symptoms rather than side effects. Unfortunately, diagnostic guidelines require the presence of nausea and/or vomiting and phonophobia and photophobia as accompanying symptoms in migraine attacks. There is no attention on other symptoms and therefore they are not recorded as such during clinical trials. Instead, they are considered as drug adverse events. Patients should be preliminarily asked during clinical interview to describe any other symptoms, although not required for diagnosis, in order to subtract them from those occurring after medication. Enriching clinical trials with this information may help for better choice of treatment and better definition of adverse events and accompanying symptoms as well.

The analysis proposed by Belsey [14], although declared as sponsored by an educational grant by a pharmaceutical company, provides analytic data from 28 efficacy studies and 35 tolerability studies (25 studies are coincident) that were chosen according to a validity index. All studies were randomised, placebo-controlled and double blind. Data are expressed as TG, TP, NNT and NNH values. The information obtained are comparable to those from the meta-analysis by Ferrari et al. [12]. As Belsey states, "this kind of analysis provides a clinically intuitive treatment choice and the methodology may be applied to different therapeutic areas". The method offers the opportunity of tailoring therapy to any single patient according to his/her needs, expectations and preferences (Fig. 2) [15]. However, in Tfelt-Hansen's opinion, although comprehensive, meta-analyses have the limitation of different data sources, well performed head-to-head comparison studies being the best scientific approach to obtain valuable clinical data [16].

\section{References}

1. Sackett DL, Richardson WS, Rosenberg WM, Haynes BR (1999) Evidence-based medicine. How to practice and teach EBM. Churchill Livingstone, New York

2. Sheftell FD, Tepper SJ (2002) New paradigms in the recognition and acute treatment of migraine. Headache 42:58-69

3. Headache Classification Committee of the International Headache Society (1988) Classification and diagnostic criteria for headache disorders, cranial neuralgias and facial pain. Cephalalgia 8[Suppl 7]:1-96

4. Pilgrim AJ (1991) Methodology of clinical trials of sumatriptan in migraine and cluster headache. Eur Neurol 31:295-299

5. International Headache Society Clinical Trial Subcommittee (2000) Guidelines for controlled trials of drugs in migraine, second edition. Cephalalgia 20:765-786

6. Mathew NT, Kailasam J, Meadors L (2004) Early treatment of migraine with rizatriptan: a placebo-controlled study. Headache 44:699-673
7. Dodick DW (2003) A review of the clinical efficacy and tolerability of almotriptan in acute migraine. Expert Opin Pharmacother 4:1157-1163

8. Ryan R, Geraud G, Goldstein J, Cady R, Keywood C (2002) Clinical efficacy of frovatriptan: placebo-controlled study. Headache 42[Suppl 2]:S84-S92

9. Geraud G, Spierings ELH, Keywood C (2002) Tolerability and safety of frovatriptan with short-and long-term use for treatment of migraine and in comparison with sumatriptan. Headache 42[Suppl 2]:S93-S99

10. Tfelt-Hansen P, Steiner T (2003) The truth about frovatriptan. Headache 43:699-700

11. Lipton RB, Pascual J, Goadsby PJ, Massiou H, McCarroll KA, Vandormael K, Jiang K, Lines CR (2001) Effect of rizatriptan and other triptans on the nausea symptom of migraine: a post hoc analysis. Headache 41:754-763
12. Ferrari MD, Roon KI, Lipton RB, Goadsby PJ (2001) Oral triptans (serotonin 5-HT1B/1D agonists) in acute migraine treatment: a meta-analysis of 53 trials. Lancet 358:1668-1675

13. Buzzi MG, Fabbri L (2000) New methods for evaluating acute treatments in migraine. Funct Neurol 15[Suppl 3]:192-195

14. Belsey J (2001) Reconciling effectiveness and tolerability in oral triptan therapy: a quantitative approach to decision making in migraine management. J Clin Res 4:105-125

15. Lanteri-Minet M (2005) What do patients want from their acute migraine therapy? Eur Neurol 53[Suppl 1]:3-9

16. Tfelt-Hansen $P$ (2006) Understanding clinical trials in migraine. $J$ Headache Pain 7:101-108 